\title{
A New Concept for the Exploration of Europa
}

\author{
Pabulo Henrique Rampelotto
}

\begin{abstract}
The Europa Jupiter System Mission (EJSM) is the major Outer Planet Flagship Mission in preparation by NASA. Although well designed, the current EJSM concept may present problematic issues as a Flagship Mission for a long-term exploration program that will occur over the course of decades. For this reason, the present work reviews the current EJSM concept and presents a new strategy for the exploration of Europa. In this concept, the EJSM is reorganized to comprise three independent missions focused on Europa. The missions are split according to scientific goals, which together will give a complete understanding of the potential habitability of Europa, including in situ life's signal measurements. With this alternative strategy, a complete exploration of Europa would be possible in the next decades, even within a politically and economically constrained environment. Key Words: Europa-Flagship Mission-EJSM-Mission concept-Exploration-Strategy. Astrobiology 12, 529-534.
\end{abstract}

\section{Introduction}

$\mathbf{I}$ N THE EARLY DECADES of space science, solar exploration programs were dominated by scientific missions designed to simply discover the general nature of the Solar System, which encompassed the Mariner missions to Venus, Mercury, and Mars and the Pioneer (Ulivi and Harland, 2007) and Voyager (Evens and Harland, 2003) explorations of planets and satellites in the outer Solar System. A new phase of exploration began with the Viking missions to Mars, the Magellan mission to Venus (Ulivi and Harland, 2008), the Galileo mission to Jupiter and its satellites (Harland, 2007), and the Cassini-Huygens mission to Saturn and its satellites (Harland, 2007). The goals of these missions reflected a new focus: more intensive exploration, including in situ measurements with landing and atmospheric probes.

Data returned from these missions have initiated an exciting new period of exploration, as we begin the process of learning about the vast diversity of environments that may support life. Consequently, Solar System exploration has been structured around the concept of habitability-the ability of a planet or moon to sustain life for extended periods of time or for a planetary system to host a planet or moon that can in turn sustain life (Rampelotto, 2010a). The habitability concept provides a unifying theme for an exploration program, which includes research and analysis and leads to a set of exploration objectives.

In this context, the next great step in space science will be the exploration of the outer Solar System, where planetary bodies have been emerging as potential habitable worlds (McKay et al., 2008; Pappalardo et al., 2009; Shapiro and Schulze-Makuch, 2009; Lorenz and Mitton, 2010; Rampelotto, 2010b). These environments provide an opportunity to extend the concept of planetary habitability (which was previously limited to Earth-like planets) and improve our understanding about the nature and distribution of habitable environments in the universe (Javaux and Dehant, 2010; Rampelotto, 2010a).

The complexity of such an endeavor requires significant focused technology development prior to mission start, and extended engineering developments as well as extensive pre-decisional trade studies to determine the proper balance of cost, risk, and science return. For these reasons, they represent major national, and often international, investments that must be selected and implemented in a strategic manner.

The Europa Jupiter System Mission (EJSM) is the major Outer Planet Flagship Mission currently in preparation by NASA. An advanced design has already been proposed for this mission (Blanc et al., 2009). However, due to its high complexity and cost, problematic issues may arise if this Flagship Mission is to be considered in the long term, that is, in conjunction with an exploration program implemented over the course of decades. In fact, the Planetary Science Decadal Survey Committee indicated that the cost for the Jupiter Europa Orbiter (JEO) as currently designed (\$4.7 billion) is so high that both a decrease in mission scope and an increase in NASA's planetary budget would be necessary

Interdisciplinary Center for Biotechnology Research, Federal University of Pampa, São Gabriel, RS—Brazil. 
to make it affordable without eliminating any other recommended mission (Committee on the Planetary Science Decadal Survey, 2011). According to the committee, NASA should undertake an effort to find major cost reductions to JEO. With these potential problematic issues, which could delay or even put an end to the current EJSM framework, alternative concepts should be considered that would have the capacity to eliminate these problems and achieve all the mission goals without compromising parallel and future missions.

It is important to consider that, even when a particular mission is well understood and planned, technical problems may demand significant changes or modifications prior to final design and launch, which can result in significant budget growth [e.g., the Mars Science Laboratory project (Lawler, 2008; Committee on Cost Growth in NASA Earth and Space Science Missions, 2010)]. In the future, these problematic issues may arise in a climate of enormous political and financial stress, which could aggravate the impact that the schedule and cost growth of one mission would have on the entire exploration program. This scenario may be the case for outer Solar System exploration, which involves expensive and complex missions for the coming decades. For this reason, the present work reviews the current EJSM concept and presents a new strategy for the exploration of Europa. With this alternative strategy, a complete exploration and understanding of Europa would be possible in a few decades, even within a politically and economically constrained environment.

\section{A Mission to Europa-Ganymede-the EJSM concept}

The EJSM concept (ESA working title: Laplace) consists of two primary flight elements operating in the jovian system: the NASA-led Jupiter Europa Orbiter (JEO) and the ESA-led Jupiter Ganymede Orbiter (JGO). JEO and JGO are designed to execute a choreographed exploration of the jovian system before settling into orbit around Europa and Ganymede, respectively (EJSM Final Report, 2009). The EJSM is guided by the overarching theme: The emergence of habitable worlds around gas giants. Detailed architectural studies for this mission over the past decade have resulted in an advanced Flagship concept (Blanc et al., 2009; Clark et al., 2011). However, the current EJSM concept still presents some issues worthy of discussion by the scientific community.

It is important to note that the europan surface is exposed to one of the harshest radiation environments in the Solar System (5400 mSv/day) (Paranicas et al., 2007). This very high level of radiation from high-energy charged particles trapped in Jupiter's magnetosphere can dissociate any organic molecules on the surface and may have a similar effect on prebiotic chemistry emerging from the interior. Hence, the presence of biosignatures on the surface of this moon seems not feasible, or at least their concentration may be very low (Rampelotto, 2010b).

Therefore, the astrobiological interest on Europa lies in the possible existence of life inside the internal ocean and biosignatures in a subsurface environment (Abbas and Schulze-Makuch, 2008; Pappalardo et al., 2009; Rampelotto, 2010c). Before sending a lander probe or penetrator to Eu- ropa, it is necessary to define astrobiologically interesting landing sites, where liquid water from the ocean could have recently reached the surface or near surface. Whether large cracks represent these locales remains controversial, and it is still unclear how to interpret features on Europa's surface (Spencer, et al., 2006). On the basis of current knowledge, it would be difficult to determine with confidence where potential landing sites may be. It would first be necessary to analyze results from studies of the ice crust and its thickness along with high-resolution images of other geological features. Without a better understanding of Europa, sending in situ instruments there would be premature and highly risky.

In this context, the proposed next Flagship Mission to Europa would be an orbiter restricted to producing images in various wavelengths as proposed for JEO. The data would bring some closure to arguments about the thickness of the crust and the existence of an aqueous internal ocean, and could identify places where the subsurface can be more easily accessed. However, in the context of a direct search for signs of life, such an orbiter would only be a precursor to the next mission, which would be probes that are able to access the subsurface and internal ocean and provide in situ life signal measurements. Considering the complexity and cost of a mission to the outer Solar System along with the arguments previously discussed, such a mission to detect life or biosignatures on Europa may not be feasible for decades to come.

Though new technology development would not be required for a mission to Europa, it would be necessary to adapt current instrument designs into a single orbiter with the capacity to perform within a harsh radiation environment. Radiation poses a unique technical challenge for the EJSM in that the flight system would have to endure a significant amount of time in the jovian radiation belts. While Galileo spent the equivalent of several weeks in the jovian magnetosphere, JEO, as currently envisioned, would have to function in this harsh radiation environment more than five times longer (JPL, 2008a). The radiation dose level, transient noise, and dose rate effects experienced by JEO would be unprecedented for long-duration NASA missions. Exposure to these large dose rates would present challenges for the science instruments even with localized shielding. High radiation fluxes create radiation noise that degrades the performance of detectors in science instruments and star trackers (Clark et al., 2009). Analysis of radiation dose, including the Europa shadowing effect, has indicated a total mission ionizing dose estimate of 2.9 Mrad behind 100 mils $(2.5 \mathrm{~mm})$ of aluminum (JPL, 2006). Although extensive work has been performed at NASA in the last decade, advances in electronic and instrument concepts will need to be made to ensure survival at the expected dose of radiation (JPL, 2008b).

With this considerable challenge, which could delay or even put an end to the current EJSM framework, an alternative Europa Mission concept should be investigated such that it would have the capability to eliminate problems with the radiation environment and achieve all scientific goals without compromising parallel and future missions.

The JEO mission is one component of the EJSM. The European-led component of the EJSM is an orbiter designed to investigate Ganymede (Jupiter Ganymede Orbiter, JGO), 
which is considered a high-priority target for astrobiology (Blanc et al., 2009). The habitability of this planetary body, however, is based solely on the presence of water. Considering that the subsurface ocean on Ganymede is probably sandwiched between two layers of ice and the subsurface ice crust may measure $100 \mathrm{~km}$ or more in thickness, the existence of sources capable of sustaining chemical complexity and effective sources of energy is highly improbable (Rampelotto, 2010d). For these reasons, Ganymede seems to have little capacity to sustain life, and consequently its astrobiological potential is quite low.

Nevertheless, due to Ganymede's interesting features (e.g., the presence of a weak magnetic field), it should be a highpriority target for planetary science, and a future mission to this satellite is worthy of consideration. In this context, EJSMLaplace is being reformulated by ESA as a European-led single spacecraft mission to the jovian system. The concept has been recently renamed JUICE (JUpiter ICy moon Explorer) and is based on the design of JGO (Dougherty et al., 2011).

\section{An Alternative Mission Concept to Europa}

The considerations outlined above suggest that some issues concerning the current EJSM concept remain open for discussion. For example, radiation represents a critical challenge that may delay and even put and end to the mission, which would further compromise the development of future missions to the outer Solar System. Also, the current design of the EJSM would not be sufficient to fully address the habitability of Europa, which will likely take many decades to be realized; furthermore, Ganymede must be considered a low priority in terms of astrobiological potential.

For this reason, an alternative project for the EJSM was designed. This project splits the Europa Flagship Mission into three smaller missions. Although the operation and launch vehicle budgets increase with three distinctly separate smaller missions, individual missions are more focused and require less costly spacecraft, instruments, and operations. This new framework affords several advantages that are worthy of consideration. The main advantage is complete access to the habitability of Europa in that the third mission would include two penetrators (or a lander probe) that would provide in situ life signal measurements. Also, the radiation hardening steps for these missions would be much simpler and incur less cost risk than those required for JEO. Lower radiation, mass, environmental, and resource requirements on the spacecraft electronics and instruments would reduce one of the largest potential cost and technical risks to the mission. Reduced radiation and mass challenges would require fewer costly technical maturation programs and redesigns of heritage subsystems and instruments. This would reduce technical, programmatic, and cost risk. Since new developments in technology are not required for this proposed strategy, the first mission could be launched during this decade, and initial results could be obtained by the mid 2020s. The general concept of each one of the three missions is described below.

\subsection{Mission 1}

Mission 1 would be an orbital spacecraft with two science objectives: to characterize Europa's ocean and its ice shell. It would answer many primary questions, such as the following: Does a subsurface ocean exist, and if so, what are its physical properties? How does the ocean circulate and interact with the silicate interior? Is the silicate seafloor active with the existence of cryovolcanism? What is the thickness of the ice shell, and how might it vary locally, regionally, and globally? The instruments required for such an endeavor would include a laser altimeter, a radio science subsystem, a microgradiometer, a magnetometer, and ice-penetrating radar. Through the laser altimeter it is possible to obtain direct proof of the existence of a liquid layer by measuring the amplitude of surface motions due to tidal forcing. Gravimetric measurements could offer independent direct proof. Accurate altimetry measurements and the precise determination of the gravity field at different altitudes would infer some constraints about the topography and/or mass anomalies at the silicate core/ liquid interface. The microgradiometer would be essential for detection of medium-scale features $(200 \mathrm{~km})$ on the seafloor, such as large volcanoes or long ridges, by obtaining high-resolution gravity field information. The magnetometer could analyze in detail the induction response from Europa's ocean. The ice-penetrating radar could provide direct constraints on the icy crust thickness and identify the ice/water interface and warm ice or water pockets within the ice shell. All these data could, in the end, be used to model the internal structure, which would greatly improve our understanding of the evolution and dynamics of this planetary body.

\subsection{Mission 2}

Mission 2 would be an orbital spacecraft with two science objectives: to characterize Europa's surface morphology and dynamics, and to determine its surface composition and chemistry. The instruments for this mission would include a narrow-angle camera to record local-scale geological processes and a medium- and wide-angle camera to record regional scale processes and morphology. A thermal imager would be included to map temperature anomalies and thermal inertia of surface materials on Europa that would help locate any active "hot spots." A visible-IR spectrometer and an ion and neutral mass spectrometer would be included as well to determine the chemistry of the europan surface. If resurfacing is a common occurrence, an analysis of surface composition could reveal the composition of the ocean and ice. However, due to the harsh radiation environment it is quite possible that exposed materials on the europan surface are affected and may not contain signatures from the potential habitable environment. The extent of how these materials are modified (a point of great interest) remains completely unknown but could be answered by such a mission.

The results from Mission 2 would have considerable importance for the understanding of geological processes on Europa, and if combined with the results from the first mission, a better understanding of the coupling between the europan ocean and its icy crust and of ocean/surface interaction would be provided. Furthermore, geological mapping at mid to high resolution combined with spectroscopic data would be used to help select potential astrobiologically significant landing sites in areas where non-ice components are 
present in large enough quantities to be easily detected and studied by a lander or penetrator.

\subsection{Mission 3}

Mission 3 would be an orbital spacecraft carrying in situ instruments with two science objectives: to characterize potential astrobiological environments and to search for biosignatures. This mission would address science objectives that could only be performed from the surface. Since the presence of complex molecules on the surface of Europa may be very constrained due to the high doses of radiation, a penetrator would best reveal the in situ composition of the subsurface environment and its physical properties and determine how the physical-chemical properties vary with depth. Furthermore, while orbital data acquisition could survey the astrobiological potential of the global surface for organic and inorganic biosignatures, only a subsurface probe could truly perform definitive direct detection of life or its biosignatures. In addition, some geophysical data (e.g., seismic measurements, surface heat flow, and magnetic field measurements) could only be precisely performed from the surface, and other measurements, such as in situ chemistry of the ocean layer and ice crust, would require a penetrator.

For this reason, several international institutions and consortiums have expressed interest in establishing investigations on Europa with the use of penetrators, with some instruments developed and tested in recent years (Gao et al., 2007; ESA, 2009; Kaufmann et al., 2009; Smith et al., 2010). In this context, Mission 3 would be devoted to delivering two penetrators on Europa. The low-mass $(5-15 \mathrm{~kg}$ ) probes considered as well as their robust design and reduced radiation shell (since they operate in the subsurface) are some factors that would make them low-cost options as opposed to surface landers (Gowen et al., 2011).

Each penetrator would carry a suite of instruments that would facilitate key astrobiology investigations as well as contribute to our understanding of the geophysics and the overall environment. Once impacted, the penetrator could release a system to explore the shallow subsurface of Europa. The sampling system could be a thermal drill, which is a combination of a rotary drill and a melting probe (Weiss et al., 2008). Such a hybrid locomotion mechanism would be advantageous in terms of penetration performance, sample acquisition, and mission security. The hybrid system (rotary drill and melting probe) in combination with a high-velocity penetrator could reach depths of up to $10 \mathrm{~m}$, which is far beyond the depths that could be reached by a penetrator $(\sim 1 \mathrm{~m})$ or a drilling instrument $(\sim 2 \mathrm{~m})$ (Weiss et al., 2011). The advantage of a thermal drill in comparison to a melting probe is that it can penetrate through the impurities (i.e., regolith and rock) that can be expected in planetary ice layers. Two penetrators would provide a variety of complementary samples, enhance the chances of finding biosignatures, and improve our understanding of the europan environment. The landing sites would be defined according to data and analysis from Missions 1 and 2.

Although penetrators have advantages regarding surface landers, it is important to point out that delivering them at Europa would be very difficult and risky. A number of critical issues still need to be developed at the earliest stages of the project. For this reason, the development of surface landers remains an important option for in situ life-signal measurements at Europa. Several lander missions have been considered during the past decade, and an advanced design has already been proposed and continues to be improved (Korablev et al., 2011; Zelenyi et al., 2011). A lander would carry more payload instruments and have a significantly longer lifetime when compared to smaller devices such as penetrators. A lander project would capitalize on existing technologies and landing systems that have already passed a full cycle of testing, which would reduce risks during the landing on Europa's surface. To access the subsurface environment, the lander could reasonably accommodate a melting probe with a mass of $\sim 5 \mathrm{~kg}$, including the payload (Biele et al., 2011).

The high mass and budget of a lander (e.g., the Russian Laplace-Europa lander with a total mass of $1210 \mathrm{~kg}$ ) remains a serious problem that could compromise the development of a lander mission for Europa. For this reason, an effort should be made to keep the cost for a lander within the New Frontiers Missions concept. One way to accomplish this would be to reduce the duration of the mission. Instead of performing measurements for 2 months, as has originally been proposed, a future lander could achieve its science objectives in 1 or 2 weeks. This strategy would significantly reduce the shielding necessary to protect the instruments from the harsh radiation of Europa and, hence, significantly reduce the cost of the mission.

\section{Concluding Remarks}

Although well designed, the current EJSM concept may present problematic issues when this Flagship Mission is considered within a long-term exploration program over the course of decades. For this reason, the present work brings into discussion the current EJSM concept and presents a new strategy for the exploration of Europa.

In this concept, the EJSM is reorganized into three independent missions focused on Europa. The missions are split according to scientific goals, which, together, would give a complete understanding of the potential habitability of Europa, including in situ life's signal measurements. Radiation challenges would be significantly reduced, and the initial results from the first mission would be acquired much earlier than they would be with the EJSM. Furthermore, allowing for budget phasing and flexibility that is lower risk and much more achievable would reduce the impact on other planetary and space science programs that would result from EJSM cost and growth. The concept of each of the three missions was developed according to science objectives, such that the results from the early mission would influence the design of those that would come later. However, other combinations should be studied in detail to realize a better distribution of the payload mass and budget.

To conclude, rather than a discussion of detailed studies for an individual mission, this work presents general concepts from a new point of view. The underlying intent is to provoke healthy debate within the scientific community toward the continuous development of a comprehensive and integrated strategy for the exploration of our solar system. 


\section{Acknowledgments}

The author would like to thank Christopher P. McKay, Robert Pappalardo, and Dirk Schulze-Makuch, whose enlightening comments significantly improved the manuscript.

\section{Abbreviations}

EJSM, Europa Jupiter System Mission; JEO, Jupiter Europa Orbiter; JGO, Jupiter Ganymede Orbiter.

\section{References}

Abbas, S.H., and Schulze-Makuch, D. (2008) Amino acid synthesis in Europa's subsurface environment. Int J Astrobiology 7:193-203.

Biele, J., Ulamec, S., Hilchenbach, M., and Komle, N.I. (2011) Insitu analysis of Europa ices by short-range melting probes. Adv Space Res 48:755-763.

Blanc, M., Alibert, Y., André, N., Atreya, S., Beebe, R., Benz, W., Bolton, S.J., Coradini, A., Coustenis, A., Dehant, V., Dougherty, M., Drossart, P., Fujimoto, M., Grasset, O., Gurvits, L., Hartogh, P., Hussmann, H., Kasaba, Y., Kivelson, M., Khurana, K., Krupp, N., Louarn, P., Lunine, J., McGrath, M., Mimoun, D., Mousis, O., Oberst, J., Okada, T., Pappalardo, R., Prieto-Ballesteros, O., Prieur, D., Regnier, P., Roos-Serote, M., Sasaki, S., Schubert, G., Sotin, C., Spilker, T., Takahashi, Y., Takashima, T., Tosi, F., Turrini, D., van Hoolst, T., and Zelenyi, L. (2009) LAPLACE: a mission to Europa and the Jupiter System for ESA's Cosmic Vision Programme. Experimental Astronomy 23:849-892.

Clark, K., Yan, T.Y., Boldt, J., Van Houten, T., Jun, I., McClure, S., and Rasmussen, R. (2009) Radiation challenges and risk mitigation for the JEO mission [paper 208576]. In Proceedings of Nuclear and Emerging Technologies for Space, the American Nuclear Society and the American Institute of Aeronautics and Astronautics.

Clark, K., Boldt, J., Greeley, R., Hand, K., Jun, I., Lock, R., Pappalardo, R., van Houten, T., and Yan, T. (2011) Return to Europa: overview of the Jupiter Europa orbiter mission. Adv Space Res 48:629-650.

Committee on Cost Growth in NASA Earth and Space Science Missions. (2010) Controlling Cost Growth of NASA Earth and Space Science Missions, The National Academies Press, Washington DC.

Committee on the Planetary Science Decadal Survey. (2011) Vision and Voyages for Planetary Science in the Decade 2013-2022, The National Academies Press, Washington DC.

Dougherty, M.K., Grasset, O., Bunce, E., Coustenis, A., Titov, D.V., Erd, Ch., Blanc, M., Coates, A.J., Coradini, A., Drossart, P., Fletcher, L., Hussmann, H., Jaumann, R., Krupp, N., Prieto-Ballesteros, O., Tortora, P., Tosi, F., Van Hoolst, T., and Lebreton, J.-P. (2011) JUICE (JUpiter ICy moon Explorer): a European-led mission to the Jupiter system [abstract 1343-1]. In EPSC-DPS Joint Meeting 2011, European Planetary Science Congress and the Division for Planetary Sciences.

EJSM Final Report. (2009, February 12) Available online at http:// opfm.jpl.nasa.gov/files/JEO-Rpt_Public-Release_090203.pdf.

ESA. (2009) Penetrator development within framework of a jovian moon mission-phase 1, ESA Ref. C213-001PA, European Space Agency, Paris.

Evens, B. and Harland, D.M., editors. (2003) NASA's Voyager Missions: Exploring the Outer Solar System and Beyond, Springer, Berlin.
Gao, Y., Ellery, A., Jaddou, M., Vincent, J., and Eckersley, S. (2007) Planetary micro-penetrator concept study with a biomimetric drill and sampler design. IEEE Trans Aerosp Electron Syst 43:875-885.

Gowen, R.A., Smith, A., Fortes, A.D., Barber, S., Brown, P., Church, P., Collinson, G., Coates, A.J., Collins, G., Crawford, I.A., Dehant, V., Chela-Flores, J., Griffiths, A.D., Grindrod, P.M., Gurvits, L.I., Hagermann, A., Hussmann, H., Jaumann, R., Jones, A.P., Joy, K.H., Karatekin, O., Miljkovic, K., Palomba, E., Pike, W.T., Prieto-Ballesteros, O., Raulin, F., Sephton, M.A., Sheridan, S., Sims, M., Storrie-Lombardi, M.C., Ambrosi, R., Fielding, J., Fraser, G., Gao, Y., Jones, G.H., Kargl, G., Karl, W.J., Macagnano, A., Mukherjee, A., Muller, J.P., Phipps, A., Pullan, D., Richter, L., Sohl, F., Snape, J., Sykes, J., and Wells, N. (2011) Penetrators for in situ subsurface investigations of Europa. Adv Space Res 48:725-742.

Harland, D.M., editor. (2007) Cassini at Saturn: Huygens Results, Springer, Berlin.

Javaux, E.J. and Dehant, V. (2010) Habitability: from stars to cells. Astronomy and Astrophysics Review 18:383-416.

JPL. (2006) Europa explorer radiation issue report. JPL D-34103, April 4, 2006, Jet Propulsion Laboratory, Pasadena, CA.

JPL. (2008a) JEO radiation design guidelines. JPL D-48258, October 14, 2008, Jet Propulsion Laboratory, Pasadena, CA.

JPL. (2008b) Assessment of radiation effects on science and engineering detectors for the JEO mission study. JPL D-48256, November 3, 2008, Jet Propulsion Laboratory, Pasadena, CA.

Kaufmann, E., Kargl, G., Kömle, N.I., Steller, M., Hasiba, J., Tatschl, F., Ulamec, S., Biele, J., Engelhardt, M., and Romstedt, J. (2009) Melting and sublimation of planetary ices under low pressure condition: laboratory experiments with a melting probe prototype. Earth Moon Planet 105:11-29.

Korablev, O., Gerasimov, M., Lebreton, J.-P., Hand, K., Webster, C., and Weiss, P. (2011) Strategies of Europa physics and environment characterization, methods and instruments, samples preparation. Adv Space Res 48:702-717.

Lawler, K. (2008) Rising costs could delay NASA's next mission to Mars and future launches. Science 321:1754.

Lorenz, R. and Mitton, J. (2010) Titan Unveiled: Explored. Princeton University Press, New Jersey.

McKay, C.P., Porco, C.C., Altheide, T., Davis, W.L., and Kral, T.A. (2008) The possible origin and persistence of life on Enceladus and detection of biomarkers in the plume. Astrobiology 8:909-919.

Pappalardo, R.T., McKinnon, W.B., and Khurana, K., editors. (2009) Europa, Univeristy of Arizona Press, Tucson.

Paranicas, C., Mauk, B.H., Khurana, K., Jun, I., Garrett, H., Krupp, N., and Roussos, E. (2007) Europa's near-surface radiation environment. Geophys Res Lett 34:L15103.

Rampelotto, P.H. (2010a) Planetary habitability in our solar system [abstract P31B-02]. Eos 91(26), Meeting Supplement.

Rampelotto, P.H. (2010b) The search for habitable worlds in the Solar System [abstract B06-0004-10]. In 38 ${ }^{\text {th }}$ COSPAR Scientific Assembly, Committee on Space Research, Paris.

Rampelotto, P.H. (2010c) Resistance of microorganisms to extreme environmental conditions and its contribution to astrobiology. Sustainability 2:1602-1623.

Rampelotto, P.H. (2010d) The search for life on other planets: sulfur-based, silicon-based, ammonia-based life. In Origins, Abiogenesis and the Search for Life, edited by Russell, M., Cosmology Science Publishers, Cambridge.

Shapiro, R. and Schulze-Makuch, D. (2009) The search for alien life in our solar system: strategies and priorities. Astrobiology 9:335-343. 
Smith, A., Gowen, R.A., Rees, K., Theobald, C., Brown, P., Pike, W.T., Hopf, T., Kumar, S., Church, P., Gao, Y., Jones, A., Joy, K.H., Crawford, I.A., Sheridan, S., Hagermann, A., Barber, S.J., Ball, A.J., and Wells, N. (2010) Application of penetrators within the Solar System. Advances in Geosciences 19:PS307.

Spencer, J.R., Grundy, W.M., Dumas, C., Carlson, R.W., McCord, T.B., Hansen, G.B., and Terrile, R.J. (2006) The nature of Europa's dark non-ice surface material: spatially-resolved high spectral resolution spectroscopy from the Keck telescope. Icarus 182:202-210.

Ulivi, P. and Harland, D.M., editors. (2007) Robotic Exploration of the Solar System: Part I: The Golden Age 1957-1982, Springer, Berlin.

Ulivi, P. and Harland, D.M., editors. (2008) Robotic Exploration of the Solar System: Part 2: Hiatus and Renewal, 1983-1996, Springer, Berlin.

Weiss, P., Yung, K.L., Ng, T.C., Kömle, N., Kargl, G., and Kaufmann, E. (2008) Study of a melting drill head for the exploration of subsurface planetary ice layers. Planet Space Sci 56:1280-1292.

Weiss, P., Yung, K.L., Kömle, N., Ko, S.M., Kaufmann, E., and Kargl, G. (2011) Thermal drill sampling system onboard high- velocity impactors for exploring the subsurface of Europa. Adv Space Res 48:743-754.

Zelenyi, L.M., Korablev, O., Martynov, M., Popov, G.A., Blanc, M., Lebreton, J.P., Pappalardo, R., Clark, K., Fedorova, A., Akim, E.L., Simonov, A.A., Lomakin, I.V., Sukhanov, A., and Eismont, N. (2011) Europa lander mission and the context of international cooperation. Adv Space Res 48:615-628.

Address correspondence to: Pabulo Henrique Rampelotto Interdisciplinary Center for Biotechnology Research Federal University of Pampa Antônio Trilha Avenue P.O. Box 1847 97300-000 São Gabriel, RS Brazil

E-mail: pabulo@lacesm.ufsm.br

Submitted 9 October 2011 Accepted 26 February 2012 